\title{
Relationship between asymmetrical jump-landing impact and quadriceps strength after unilateral anterior cruciate ligament reconstruction
}

\author{
Junya Aizawa $^{1 *}$, Shunsuke Ohji ${ }^{1}$, Kenji Hirohata ${ }^{1}$, Takehiro Ohmi ${ }^{1}$, Hideyuki Koga ${ }^{2}$ and Kazuyoshi Yagishita ${ }^{1}$ \\ ${ }^{1}$ Clinical Center for Sports Medicine and Sports Dentistry, Tokyo Medical and Dental University, 1-5-45 Yushima, Bunkyo-ku, Tokyo 113-8519, Japan \\ ${ }^{2}$ Department of Joint Surgery and Sports Medicine, Graduate School of Medical and Dental Sciences, Tokyo Medical and Dental University, 1-5-45 Yushima, \\ Bunkyo-ku, Tokyo 113-8519, Japan
}

\begin{abstract}
Background: The magnitude of the peak landing impact force, the rapid timing of landing, and the asymmetrical forces between limbs pose a re-injury risk after unilateral anterior cruciate ligament (ACL) reconstruction. However, the correlation between quadriceps strength asymmetry with asymmetrical jump-landing impact has not been investigated.
\end{abstract}

Objective: To examine the relationship between asymmetrical landing impact and quadriceps strength in athletes participating in jump-landing sports after unilateral ACL reconstruction.

Methods: Thirty-four athletes who had unilateral ACL reconstruction and were cleared to participate in jump-landing sports were investigated. The participants were subdivided into two groups: low quadriceps strength group ( $\mathrm{n}=15$, quadriceps strength LSI $<85 \%$ ) and high quadriceps strength group ( $\mathrm{n}=15$, quadriceps strength LSI $\geq 90 \%$ ). The magnitude of the peak vertical ground reaction force (pVGRF) and the time from initial contact to the pVGRF during single-leg landing after anterior jump were measured. Loading rate was calculated, and the LSIs of the vertical ground reaction force variables between the two groups were compared.

Results: In the low quadriceps strength group, the loading rate LSI was 114\%, which was significantly higher than that in the high quadriceps strength group $(\mathrm{P}=0.04)$, and the $\mathrm{pVGRF}$ of the of the ACL-reconstructed limb tended to be higher than that in the non-ACL-reconstructed limb ( $\mathrm{P}=0.07)$.

Conclusion: As the loading rate asymmetry was significantly greater in athletes with lower quadriceps strength LSI, evaluating the loading rate LSI in addition to quadriceps strength asymmetry when determining an athlete's physical readiness to return to sports should be considered.

\section{Introduction}

Primary and secondary injuries of the anterior cruciate ligament (ACL) often occur when athletes try to decelerate the body's momentum during single-leg landing after jumping in sports such as basketball and handball $[1,2]$. To return to sports, $80-90 \%$ of athletes who experience primary ACL injury undergo reconstruction surgery and long-term postoperative rehabilitation [3-5]. The incidence of secondary injuries for the first 2-15 years after return to sports is $5.9-34 \%$ higher than that of primary injuries [6-9]. Moreover, athletes need a longer period of time to return to sports after revision reconstruction than after primary reconstruction [10].

In cadaveric studies, ACL strain is increased by femorotibial joint compression due to the posterior slope of the tibial plateau [11-13]. During single-leg landing after anterior jumping, ACL strain measured using a strain gauge device implanted in the intact ACL reaches a peak that corresponds to the peak vertical ground reaction force (pVGRF) [14]. Therefore, the pVGRF magnitude and the loading rate, which is calculated as the pVGRF magnitude divided by the time from foot contact to the pVGRF, have been identified as the re-injury risk factors in athletes after primary reconstruction [15-18].

Myer et al. analyzed the asymmetry of the pVGRF magnitude in the landing phase during single-leg vertical jumping in athletes who returned to sports after primary/unilateral ACL reconstruction [19]. They suggested that the ACL-reconstructed (ACLR) limb has a lower absorption of the landing impact than the non-ACLR limb.

After unilateral reconstruction, internal knee extension moment during single-leg landing by the ACLR limb is lower than that by the non-ACLR limb $[18,20]$. Compared to healthy subjects, subjects who have undergone ACL reconstruction show a lower moment and lower energy absorption of the knee during single-leg landing [21]. During single-leg landing, increasing the internal moment by increasing quadriceps strength is necessary to reduce the speed and absorb the energy of the body center of mass [21].

After unilateral reconstruction in athletes, isokinetic quadriceps strength tends to be lower in the ACLR limb than in the non-ACLR limb $[18,20]$. The limb symmetry index (LSI) of quadriceps strength,

${ }^{\star}$ Correspondence to: Junya Aizawa, Clinical Center for Sports Medicine and Sports Dentistry, Tokyo Medical and Dental University, 1-5-45 Yushima, Bunkyo-ku, Tokyo 113-8519, Japan, E-mail: aizawa.spt@tmd.ac.jp

Key words: knee torque, one-leg jump, ground reaction force, side-to-side difference, return to sports

Received: July 04, 2019; Accepted: July 10, 2019; Published: July 15, 2019 
which was calculated by dividing the ACLR limb value by the nonACLR limb value and multiplying the quotient by 100 , is widely used as a criterion to judge the timing of return to sports after ACL reconstruction $[18,22,23]$.

The purpose of this study was to examine the relationships between asymmetries of quadriceps strength and the pVGRF variables during single-leg landing following jump in athletes who had undergone primary/unilateral ACL reconstruction. For this analysis, the LSIs of the pVGRF magnitude and loading rate were compared between two groups: the low quadriceps strength (LQ) group $(\mathrm{LSI}<85 \%)$ and the high quadriceps strength (HQ) group (LSI $\geq 90 \%)[18,24]$. We hypothesized that the LSIs of the pVGRF magnitude and loading rate in the LQ group would be $>100 \%$ and larger than those in the HQ group.

\section{Methods}

The Institutional Review Board at our institution approved the study design (approval number: M2016 271). All participants provided written, informed consent prior to participating in this study.

\section{Participants}

The inclusion criteria for participants after ACL reconstruction were as follows: age $\geq 16$ years and $\leq 40$ years at measurement primary/ unilateral ACL injury; underwent primary/unilateral anatomical double-bundle reconstruction using a hamstring tendon autograft; underwent postoperative rehabilitation within the same protocol discussed in the Postoperative rehabilitation section; $>150$ days after surgery; and participating in training or jump landing sports matches including basketball, handball, soccer, volleyball, badminton, tennis, lacrosse, and flying disk sports. Participants with a history of injuries or surgery of the hip, ankle, and lumbar region were excluded.

\section{Postoperative rehabilitation}

The postoperative rehabilitation protocol was the same for all patients, and the protocol did not vary with surgical meniscus treatment. Patients were permitted to begin isometric quadriceps exercises as tolerated immediately after ACL reconstruction. Using knee brace (Straighted Position Knee-Joint Immobilizer, ALCARE, Tokyo, Japan) and crutches, $20 \mathrm{~kg}$ of partial weight bearing was allowed at postoperative day 1 , which was gradually increased to the maximum body weight of each patient. Range-of-motion exercise from full extension to $120^{\circ}$ of flexion was started at postoperative day at postoperative day 3 . Use of crutches was discontinued at 4 weeks. Closed kinetic chain exercises were started at 2 weeks, and open kinetic exercises such as seated knee extension using machine load, and a perturbation training program were started at 3 months after surgery. Running exercise was started at 3 months, which commenced with jogging; the running speed was gradually increased to the maximum speed for each patient. Once $80 \%$ of full-speed running was achieved, athletic exercises related to the previous sports or desired sporting activities were initiated with detailed instructions. The athletic exercises were specific to each patient, depending on the kind of sports they were previously engaged in and on their athletic level. Participating in sports matches was allowed at 6 months after surgery, as long as the patient showed no problematic symptoms in the joint and had sufficient knee isokinetic flexion/extension strength at $60 \%$ s (LSI of approximately $80 \%)$ measured by Biodex Multi-Joint Testing and Rehabilitation System (BDX-4, Biodex Medical Systems Inc., New York, New York, USA) after the specified athletic training had been accomplished.

\section{Quadriceps strength testing and subdivision of groups}

The Biodex Multi-Joint Testing and Rehabilitation System was used for evaluating the isokinetic strength of the quadriceps. To minimize compensatory movements during testing, participants were seated and secured with padded straps around the thigh, pelvis, and torso. The femoral condyle of the tested limb was aligned with the rotation axis of the torque meter. Participants performed three repetitions of submaximal knee extension/flexion to familiarize themselves with the testing motion. To determine the strength of both quadriceps, participants performed five consecutive concentric contractions at $60 \%$ s. The peak torque within the five trials was extracted and normalized by body weight. The non-ACLR limb was tested first. The intraclass correlation coefficient of the quadriceps concentric peak torque using the Biodex system was close to 0.90 , indicating a high to very high reproducibility [25].

The LSI of the quadriceps strength was calculated by dividing the ACLR limb value by the non-ACLR limb value and multiplying the quotient by 100 . The participants were subdivided into two groups based on quadriceps strength LSI (LQ group, quadriceps strength $\mathrm{LSI}<85 \%$; HQ group, quadriceps strength $\mathrm{LSI} \geq 90 \%$ ) to evaluate the effect of quadriceps strength LSI on the LSI of single-leg landing impact $[18,24]$. These cut off values were based on a previous study on ACLreconstructed athletes that indicated that a side-to-side difference in peak quadriceps strength output of $10 \%$ is considered to reflect differences in the capacity of muscle performance beyond measurement error and commonly reported quadriceps strength criterion values for return-to-sport decision-making [26-29].

\section{Single-leg jump-landing task and pVGRF variables}

All participants wore the same type of shoes with no air cushions. The participants were verbally and visually instructed regarding how to perform the task. A 20 -cm-high step was placed $60 \mathrm{~cm}$ from the center of the force plate (260AA6, Kistler Instrumente AG, Winterthur, Switzerland). The participants stood on the step on a single leg with the other knee bent, with neutral hip rotation and the arms crossed to eliminate the effect of arm movement. The participants were asked to jump forward without any intentional upward action and landed as naturally as possible on the same leg at the center of the force plate, maintaining balance for $5 \mathrm{sec}$. The participants faced forward during all jumps and landings. All participants practiced the same tasks three times with both limbs before data collection. A trial was judged unacceptable visually if the sole of the foot did not come in contact to the center of the force plate at landing, the foot moved or slid after landing, or the opposite foot touched the force plate or floor.

Ground reaction force was collected at a sampling rate of $1000 \mathrm{~Hz}$ and filtered using a low-pass Butterworth filter with a cutoff frequency of $50 \mathrm{~Hz}$ and was normalized by body weight using software (IFS-4J/3J, $\mathrm{DKH}$, Tokyo, Japan). Extracted data included the initial contact (IC) time and the peak time of vertical ground reaction force. IC was defined as the moment when the vertical ground reaction force exceeded 10 $\mathrm{N}$. The elapsed time from the IC to the pVGRF (pVGRF time) was calculated, and the loading rate was calculated as pVGRF magnitude divided by pVGRF time [16].

\section{Statistical analysis}

The sample size estimate for this study was based on a prior pilot study evaluating differences in LSI of the pVGRF variables between HQ and LQ groups. A sample size of 15 participants per group was required to achieve a power of 0.90 with an alpha level of 0.05 . Of the 
34 participants, 15 were in the LQ group, 15 were in the HQ group, and 4 had quadriceps strength LSI between $85 \%$ and $89 \%$. Normality of the distribution of each variable was determined by the ShapiroWilk normality test. Differences in descriptive data, such as age, body size, Tegner activity level scale, and days from surgery, between groups were analyzed using a non-paired t-test or the Mann-Whitney U-test. Differences in nominal descriptive data, such as sex, ACLR limb dominance, and meniscus repair, between, groups were analyzed using the chi-squared test or Fisher's exact test.

Differences in values of quadriceps strength and pVGRF variables between groups and between limbs were examined using a non-paired $\mathrm{t}$-test and paired $\mathrm{t}$-test, respectively. When even one of the two variables, i.e., quadriceps strength and pVGRF variables, showed a non-normal distribution, the Mann-Whitney U-test or the Wilcoxon signed-rank test was used to analyze the differences.

The LSIs of quadriceps strength and pVGRF variables in the LQ group were compared with those in the HQ group using a non-paired t-test or the Mann-Whitney U-test. The level of significance for all tests was set at 0.05. The Statistical Package for the Social Sciences (version 21.0; IBM Corp., New York, USA) for Windows was used for all statistical analyses.

\section{Results}

Differences in descriptive data, except Tegner activity scale, between the LQ and HQ groups between the LQ and HQ groups were not significant (Table 1). In both groups, the quadriceps strength of the ACLR limb was significantly lower than that of the non-ACLR limb (Table 2). The average quadriceps strength LSI in the LQ group was $76.4 \%$, which was significantly lower than that in the HQ group (Table 2). For the non-ACLR limb the quadriceps strength in the LQ group tended to be higher than that in the HQ group (Table 2).

In the LQ group, the PVGRF of the ACLR limb tended to be higher than that of the non-ACLR limb (Table 2), and the difference in the pVGRF LSI between groups tended to be significant (Table 2). The average LSI of the loading rate in the LQ group was $114.4 \%$, which was significantly higher than that in the HQ group; the difference in the loading rate LSI between groups was approximately 19 points (Table 2).

\section{Discussion}

In this study two groups of athletes participating in jump landing sports after primary/unilateral ACL reconstruction (LQ group, $\mathrm{LSI}<85$ $\%$; HQ group, LSI $\geq 90 \%$ ) showed that in the low LSI group, there is lower buffer capacity in the ACLR limb than in the non-ACLR limb. The loading rate LSI was significantly higher in the LQ group than in the HQ group.

During landing, internal knee extension moment via muscle contractions is necessary to control joint motion and to absorb the kinetic energy of the body [30]. Extensor muscle activation through eccentric muscle action during landing could be described as energy absorption [31]. The maximum extension torque of the knee is a predictive factor for energy absorption in the knee during double-leg drop-jump landing [32,33]. In the total support moment, which is calculated from the total of the knee, hip, and ankle internal moments during single-leg drop-jump landing, the proportion of power absorbed by the knee is significantly lower and that by the hip is significantly higher in ACL-reconstructed athletes than in healthy subjects [21]. These findings suggest that quadriceps strength is an important determinant in the mechanics of landing impact absorption among athletes after ACL reconstruction.

In this study, although the difference in quadriceps strength in ACLR limbs between groups was not significant, the quadriceps strength tended to be higher in the LQ group than in the HQ group for the non-ACLR limb. These results indicate that the inter-group difference in quadriceps strength LSI was not due to a difference in ACLR limb muscle strength, but rather due to a difference in nonACLR limb muscle strength, which could be because the function of the ACLR limb is compensated for by the non-ACLR limb; thus, the quadriceps muscle of the non-ACLR limb has a greater burden, and as an adaptation, quadriceps strength LSI is reduced [24]. These results suggested that LSI is an important index of quadriceps strength recovery after unilateral ACL reconstruction.

Table 1. Participant characteristics

\begin{tabular}{|c|c|c|c|}
\hline & LQ (n=15) & HQ (n=15) & P value \\
\hline Age (year) & $21.5(4.0)$ & $21.0(7.0)$ & 0.803 \\
\hline Height $(\mathrm{cm})$ & $168.9(9.6)$ & $162.8(4.7)$ & 0.078 \\
\hline Weight (kg) & $62.9(12.3)$ & $58.9(7.1)$ & 0.29 \\
\hline Body mass index & $22.1(2.6)$ & $22.2(2.1)$ & 0.952 \\
\hline Sex (female ratio) & $46.70 \%$ & $73.30 \%$ & 0.136 \\
\hline Dominance of injured leg (ratio of patient number) & $53.30 \%$ & $46.70 \%$ & 0.715 \\
\hline \multicolumn{4}{|l|}{ Sports } \\
\hline Soccer & 7 & 4 & \\
\hline Volleyball & 1 & 4 & \\
\hline Basketball & 2 & 4 & \\
\hline Badminton & 3 & 2 & \\
\hline Tennis & 0 & 1 & \\
\hline Handball & 0 & 0 & \\
\hline Lacrosse & 1 & 0 & \\
\hline Flying disk sports & 1 & 0 & \\
\hline Tegner activity level scale before injury & $8.3(0.9)$ & $7.0(1.0)$ & $0.018^{*}$ \\
\hline Meniscus repair (ratio of patient number) & $60.00 \%$ & $60.00 \%$ & 0.705 \\
\hline Days from surgery to measurement & $185.0(20.7)$ & $184.9(14.5)$ & 0.984 \\
\hline Days from injury to surgery & $74.0(54.0)$ & $53.0(42.0)$ & 0.221 \\
\hline
\end{tabular}

Data are means and SD or median and interquartile range (italic). Dominant leg side was defined as the leg used to kick a ball to maximal distance.

LQ, low quadriceps strength group; HQ, high quadriceps strength group

$*$ Significance at $\mathrm{P}<0.05$. 
Table 2. Quadriceps strength and ground reaction force variables

\begin{tabular}{|c|c|c|c|c|}
\hline & LQ group $(n=15)$ & HQ group $(n=15)$ & P value between groups & Cohen's d \\
\hline \multicolumn{5}{|c|}{ Quadriceps strength (N/BW) } \\
\hline ACLR limb & $205.8(38.0)$ & $219.1(58.5)$ & 0.467 & \\
\hline Non-ACLR limb & $269.4(43.7)$ & $229.9(61.2)$ & $0.053 \dagger$ & 0.74 \\
\hline$P$ value between limbs & $<0.001^{*}$ & $0.009^{*}$ & & \\
\hline LSI & $76.4(5.6)$ & $95.5(6.1)$ & $<0.001^{*}$ & 3.26 \\
\hline \multicolumn{5}{|l|}{ pVGRF (N/BW) } \\
\hline ACLR limb & $381.6(66.3)$ & $374.1(58.2)$ & 0.744 & \\
\hline Non-ACLR limb & $363.6(62.4)$ & $382.2(39.0)$ & 0.337 & \\
\hline $\mathrm{P}$ value between limbs & $0.073 \dagger$ & 0.488 & & \\
\hline LSI & $105.6(10.3)$ & $97.8(11.7)$ & $0.065 \dagger$ & 0.71 \\
\hline \multicolumn{5}{|l|}{ pVGRF time (ms) } \\
\hline ACLR limb & $41.0(9.0)$ & $40.9(10.4)$ & 0.466 & \\
\hline Non-ACLR limb & $46.7(8.7)$ & $41.0(7.0)$ & 0.253 & \\
\hline$P$ value between limbs & 0.147 & 0.767 & & \\
\hline LSI & $92.4(22.1)$ & $102.4(35.4)$ & 0.171 & \\
\hline \multicolumn{5}{|l|}{ Loading rate (N/BW/ms) } \\
\hline ACLR limb & $8.3(2.4)$ & $9.1(5.2)$ & 0.95 & \\
\hline Non-ACLR limb & $8.0(2.1)$ & $9.5(2.6)$ & 0.165 & \\
\hline$P$ value between limbs & 0.114 & 0.175 & & \\
\hline LSI & $114.4(24.3)$ & $95.1(24.4)$ & $0.044^{*}$ & 0.79 \\
\hline
\end{tabular}

Data are means and SD or median and interquartile range (italic). Loading rate was calculated as the pVGRF divided by the elapsed time from initial contact to the $\mathrm{pVGRF}$. LSI was calculated as the ratio of the ACLR limb value divided by non-ACLR limb value. LQ, low quadriceps strength; HQ, high quadriceps strength; ACLR, anterior cruciate ligament reconstructed; $p$ GRF, peak vertical ground reaction force; LSI, limb symmetry index; N/BW, Newton per body weight

*Significance at $\mathrm{P}<0.05$.

$\dagger$ Tendency to significance at $\mathrm{P}<0.1$.

Myer et al. analyzed the asymmetry of pVGRF magnitude in the landing phase during single-leg vertical jumping in athletes who returned to sports after primary/unilateral ACL reconstruction and suggested that the ACLR limb has a lower absorption of the landing impact than the non-ACLR limb because the pVGRF magnitude normalized by jump height was larger in the ACLR limb than in the nonACLR limb [19]. Palmieri-Smith et al., in a study of ACL-reconstructed athletes who returned to sports, showed that the maximum knee flexion angle LSI during single-leg landing after anterior jumping was significantly smaller in the LQ group than in the HQ group [23]. Other previous studies showed that there is greater pVGRF with a smaller knee flexion angle during landing [34,35]. Our study's findings support those of previous studies and suggest that asymmetry of the pVGRF variables during single-leg landing should be included among the criteria for determining the timing of return to sports after primary/ unilateral ACL reconstruction.

Ithurburn et al. found no significant difference in loading rate LSI during single-leg drop-jump landing from a height of $30 \mathrm{~cm}$ between LQ and HQ groups [18]. Ali et al. examined single-leg anterior jump landing from a height of $20 \mathrm{~cm}$, analyzed the ground reaction force with different anterior distances (i.e., 30,50 , and $70 \mathrm{~cm}$ ), and showed that pVGRF and posterior ground reaction force increased with greater distance $[34,35]$. In our study, we examined landing after a $60-\mathrm{cm}$ anterior jump from a height of $20 \mathrm{~cm}$, not drop-jump landing. We found that the average loading rate LSI was approximately $114 \%$ in the LQ group and approximately $95 \%$ in the HQ group; the group difference was significant. Based on the results of previous studies and our study, landing after jumping has greater impact absorption than landing after dropping; thus, the loading rate during landing is greater in the LQ group, which has poorer energy absorbing capacity in the ACLR limb.

A stiffer landing strategy, which is associated with reduced ability of the lower extremity muscles such as the quadriceps to absorb ground reaction forces, may theoretically expose the passive anatomical structures of the knee to higher forces, thereby resulting in re-injury [30,36-38]. In this study, the results suggest that in athletes participating in sports involving jump landings with quadriceps strength LSI $<85 \%$, quadriceps strengthening focused on the ACLR limb is particularly needed even after $>5$ months since reconstruction. This finding could be used to explain to athletes and coaches that evaluating asymmetry of the loading rate and increasing buffer capacity during single-leg landing after jumping with the ACLR limb is a strategy for preventing re-injury. Moreover, this may also be used to support the importance of coaching to prevent the overuse of the non-ACLR limb to compensate for energy absorption or production in the ACLR limb in double-leg landing training.

This study has some limitations: (1) Although a sample size of 15 participants per group was required based on a prior pilot study, the effect size of group comparison for loading rate was 0.79 , which was not high. (2) The Tegner activity level was higher in the LQ group than in the HQ group; this difference possibly affected the results. (3) The data in this study were for both men and women; therefore, the characteristics of the results by sex are unknown. (4) All participants had doublebundle reconstruction using the hamstring tendon, and their results could not be simply applied to patients who had reconstruction using bone-patellar tendon bone grafts as they have different knee muscle strength characteristics [39,40]. (5) The muscle strength dynamometer in this study measured concentric muscle strength at an angular velocity of $60 \%$, and whether dividing the groups for analysis by muscle strength measured by means of a different contraction pattern or angular velocity would yield the same results remains to be established. Thus, further studies are warranted. Moreover, for a better evaluation of the buffer capacity during landing, kinematics including sagittal joint angles should be analyzed with the pVGRF variables in future studies.

\section{Conclusion}

This study suggested that asymmetry of single-leg jump-landing impact correlated with asymmetry of the quadriceps strength in athletes 
participating in jump-landing sports after primary/unilateral ACL reconstruction. Evaluation of asymmetry of landing impact, in addition to quadriceps strength asymmetry, may be essential in determining the timing of return to sports.

\section{Acknowledgments}

The authors would like to thank people involved in the translation process.

\section{Funding}

This work was supported by the JSPS KAKENHI [grant number JP26350606].

\section{Declaration of interest}

None.

\section{Conflict of interest statement}

All authors do not have any financial and personal relationships with other people or organizations that could inappropriately influence their work.

\section{References}

1. Olsen OE, Myklebust G, Engebretsen L, Bahr R (2004) Injury mechanisms for anterior cruciate ligament injuries in team handball: a systematic video analysis. Am J Sports Med 32: 1002-1012. [Crossref]

2. Krosshaug T, Nakamae A, Boden BP, Engebretsen L, Smith G, et al. (2007) Mechanisms of anterior cruciate ligament injury in basketball: video analysis of 39 cases. $\mathrm{Am} \mathrm{J}$ Sports Med 35: 359-367. [Crossref]

3. Ardern CL, Webster KE, Taylor NF, Feller JA (2011) Return to sport following anterior 7 cruciate ligament reconstruction surgery: a systematic review and meta-analysis of 8 the state of play. Br J Sports Med 45: 596-606. [Crossref]

4. Ardern CL, Webster KE, Taylor NF, Feller JA (2011) Return to the preinjury level of 10 competitive sport after anterior cruciate ligament reconstruction surgery: two thirds 11 of patients have not returned by 12 months after surgery. Am J Sports Med 39: 538543. [Crossref]

5. Linko E, Harilainen A, Malmivaara A, Seitsalo S (2005) Surgical versus conservative 14 interventions for anterior cruciate ligament ruptures in adults. Cochrane Database Syst Rev 2: CD001356. [Crossref]

6. Paterno MV, Rauh MJ, Schmitt LC, Ford KR, Hewett TE (2014) Incidence of Second ACL Injuries 2 Years After Primary ACL Reconstruction and Return to Sport. Am J Sports Med 42: 1567-1573. [Crossref]

7. Leys T, Salmon L, Waller A, Linklater J, Pinczewski L (2012) Clinica 1 results and risk 20 factors for reinjury 15 years after anterior cruciate ligament reconstruction: a 21 prospective study of hamstring and patellar tendon grafts. Am J Sports Med 40: 595-605.

8. Wiggins AJ, Grandhi RK, Schneider DK, Stanfield D, Webster KE, et al. (2016) Risk of 24 Secondary Injury in Younger Athletes After Anterior Cruciate Ligament 25 Reconstruction: A Systematic Review and Meta-analysis. Am J Sports Med 44: 18611876. [Crossref]

9. Schilaty ND, Nagelli C, Bates NA, Sanders TL, Krych AJ, et al. (2017) Incidence of Second Anterior Cruciate Ligament Tears and Identification of 29 Associated Risk Factors From 2001 to 2010 Using a Geographic Database. Orthop J Sports Med 5: 2325967117724196. [Crossref]

10. Okoroha KR, Kadri O, Keller RA, Marshall N, Cizmic Z, et al. (2017) Return to 32 Pla y After Revision Anterior Cruciate Ligament Reconstruction in National Football 33 League Players. Orthop J Sports Med 5: 2325967117698788. [Crossref]

11. Kiapour AM, Quatman CE, Goel VK, Wordeman SC, Hewett TE, Demetropoulos CK (2014) Timing Sequence of Multi-Planar Knee Kinematics Revealed by Physiologic Cadaveric Simulation of Landing: Implications for ACL Injury Mechanism. Clin Biomech (Bristol, Avon) 29: 75-82. [Crossref]

12. Markolf KL, Jackson SR, Foster B, McAllister DR (2014) ACL forces and knee kinematics produced by axial tibial compression during a passive flexion-extension cycle. J Orthop Res 32: 89-95. [Crossref]
13. Kiapour AM, Demetropoulos CK, Kiapour A, Quatman CE, Wordeman SC, et al. (2016) Strain Response of the Anterior Cruciate Ligament to Uniplanar and Multiplanar Loads During Simulated Landings: Implications for Injury Mechanism. Am J Sports Med 44: 2087-2096. [Crossref]

14. Cerulli G, Benoit DL, Lamontagne M, Caraffa A, Liti A (2003) In vivo anterior cruciate ligament strain behaviour during a rapid deceleration movement: case report. Knee Surg Sports Traumatol Arthrosc 11: 307-311. [Crossref]

15. Paterno MV, Schmitt LC, Ford KR, Rauh MJ, Myer GD, et al. (2010) Biomechanical measures during landing and postural stability predict second anterior cruciate ligament injury after anterior cruciate ligament reconstruction and return to sport. Am J Sports Med 38: 1968-1978.

16. Dai B, Butler RJ, Garrett WE, Queen RM (2014) Using ground reaction force to predict knee kinetic asymmetry following anterior cruciate ligament reconstruction. Scand $J$ Med Sci Sports 24: 974-981.

17. Decker MJ, Torry MR, Noonan TJ, Riviere A, Sterett WI (2002) Landing adaptations after ACL reconstruction. Med Sci Sports Exerc 34: 1408-1413.

18. Ithurburn MP, Paterno MV, Ford KR, Hewett TE, Schmitt LC (2015) Young Athletes with Quadriceps Femoris Strength Asymmetry at Return to Sport After Anterior Cruciate Ligament Reconstruction Demonstrate Asymmetric Single-Leg Drop-Landing Mechanics. Am J Sports Med 43: 2727-2737. [Crossref]

19. Myer GD, Martin L, Ford KR, Paterno MV, Schmitt LC, et al. (2012) No association of time from surgery with functional deficits in athletes after anterior cruciate ligament reconstruction: evidence for objective return-to-sport criteria. Am J Sports Med 40: 2256-2263. [Crossref]

20. Trigsted SM, Post EG, Bell DR (2017) Landing mechanics during single hop for distance in females following anterior cruciate ligament reconstruction compared to healthy controls. Knee Surg Sports Traumatol Arthrosc 25: 1395-1402. [Crossref]

21. Pozzi F, Di Stasi S, Zeni JA Jr, Barrios JA (2017) Single-limb drop landing biomechanics in active individuals with and without a history of anterior cruciate ligament reconstruction: A total support analysis. Clin Biomech (Bristol, Avon) 43: 2833. [Crossref]

22. Kyritsis P, Bahr R, Landreau P, Miladi R, Witvrouw E (2016) Likelihood of ACL graf rupture: not meeting six clinical discharge criteria before return to sport is associated with a four times greater risk of rupture. Br J Sports Med 50: 946-951. [Crossref]

23. Palmieri-Smith RM, Lepley LK (2015) Quadriceps Strength Asymmetry After Anterior Cruciate Ligament Reconstruction Alters Knee Joint Biomechanics and Functional Performance at Time of Return to Activity. Am J Sports Med 43: 1662-1669. [Crossref]

24. Schmitt LC, Paterno MV, Ford KR, Myer GD, Hewett TE (2015) Strength Asymmetry and Landing Mechanics at Return to Sport after Anterior Cruciate Ligament Reconstruction. Med Sci Sports Exerc 47: 1426-1434. [Crossref]

25. de Araujo Ribeiro Alvares JB, Rodrigues R, de Azevedo Franke R, da Silva BG, Pinto RS, et al. (2015) Inter-machine reliability of the Biodex and Cybex isokinetic dynamometers for knee flexor/extensor isometric, concentric and eccentric tests. Phys Ther Sport 16: 59-65. [Crossref]

26. Schmitt LC, Paterno MV, Hewett TE (2012) The impact of quadriceps femoris strength asymmetry on functional performance at return to sport following anterior cruciate ligament reconstruction. J Orthop Sports Phys Ther 42: 750-759. [Crossref]

27. Sapega AA (1990) Muscle performance evaluation in orthopaedic practice. $J$ Bone Joint Surg Am 72: 1562-1574. [Crossref]

28. Kvist J (2004) Rehabilitation following anterior cruciate ligament injury: current recommendations for sports participation. Sports Med 34: 269-280. [Crossref]

29. Roi GS, Creta D, Nanni G, Marcacci M, Zaffagnini S, et al. (2005) Return to official Italian First Division soccer games within 90 days after anterior cruciate ligament reconstruction: a case report. J Orthop Sports Phys Ther 35: 52-61. [Crossref]

30. Devita P, Skelly WA (1992) Effect of landing stiffness on joint kinetics and energetics in the lower extremity. Med Sci Sports Exerc 24: 108-115. [Crossref]

31. McNitt-Gray JL (1993) Kinetics of the lower extremities during drop landings from three heights. J Biomech 26: 1037-1046. [Crossref]

32. Schmitz RJ, Shultz SJ (2010) Contribution of knee flexor and extensor strength on sex-specific energy absorption and torsional joint stiffness during drop jumping. $J$ Athl Train 45: 445-452. [Crossref] 
Aizawa J (2019) Relationship between asymmetrical jump-landing impact and quadriceps strength after unilateral anterior cruciate ligament reconstruction

33. Montgomery MM, Shultz SJ, Schmitz RJ, Wideman L, Henson RA (2012) Influence of lean body mass and strength on landing energetics. Med Sci Sports Exerc 44: 23762383. [Crossref]

34. Ali N, Robertson DG, Rouhi G (2014) Sagittal plane body kinematics and kinetics during single leg landing from increasing vertical heights and horizontal distances: implications for risk of non-contact ACL injury. Knee 21: 38-46. [Crossref]

35. Ali N, Rouhi G, Robertson G (2013) Gender, Vertical Height and Horizontal Distance Effects on Single-Leg Landing Kinematics: Implications for Risk of non-contact ACL Injury. J Hum Kinet 37: 27-38. [Crossref]

36. Decker MJ, Torry MR, Wyland DJ, Sterett WI, Richard Steadman J (2003) Gender differences in lower extremity kinematics, kinetics and energy absorption during landing. Clin Biomech (Bristol, Avon) 18: 662-669. [Crossref]
37. Lephart SM, Ferris CM, Riemann BL, Myers JB, Fu FH (2002) Gender differences in strength and lower extremity kinematics during landing. Clin Orthop Relat Res 401: 162-169. [Crossref]

38. Zhang SN, Bates BT, Dufek JS (2000) Contributions of lower extremity joints to energy dissipation during landings. Med Sci Sports Exerc 32: 812-819. [Crossref]

39. Machado F, Debieux P, Kaleka CC, Astur D, Peccin MS, et al. (2018) Knee isokinetic performance following anterior cruciate ligament reconstruction: patellar tendon versus hamstrings graft. Phys Sportsmed 46: 30-35. [Crossref]

40. Ageberg E, Roos HP, Silbernagel KG, Thomeé R, Roos EM (2009) Knee extension and flexion muscle power after anterior cruciate ligament reconstruction with patellar tendon graft or hamstring tendons graft: a cross-sectional comparison 3 years postsurgery. Knee Surg Sports Traumatol Arthrosc 17: 162-169. [Crossref]

Copyright: (C2019 Aizawa J. This is an open-access article distributed under the terms of the Creative Commons Attribution License, which permits unrestricted use, distribution, and reproduction in any medium, provided the original author and source are credited. 